\title{
GLOBAL EXISTENCE AND UNIQUENESS FOR IMPLICIT DIFFERENTIAL EQUATION OF ARBITRARY ORDER
}

\author{
Sagar T. Sutar And Kishor D. Kucche
}

Abstract. The aim of this paper is to establish the existence result for implicit differential equation of fractional (arbitrary) order via topological transversality theorem known as Leray-Schauder alternative. Further we prove the uniqueness results. The Grownwall's lemma for singular kernels play an important role to prove our results. We verify our results by providing an example. Mathematics subject classification (2010): 26A33, 34A08.

Keywords and phrases: Implicit fractional differential equation, Leray-Schauder alternative, existence and uniqueness, integral inequality.

\section{REFERENCES}

[1] R. P. Agarwal, M. Belmekki, M. Benchohra, A survey on semilinear differential equations and inclusions involving Riemann-Liouville fractional derivative, Adv. Diff. Equ. 47 (2009), Article ID 981728.

[2] R. P. Agarwal, Yong Zhou, Yunyun He, Existence of fractional neutral functional differential equations, Computers and Mathematics with Applications, 59 (2010), 1095-1100.

[3] S. BHALEKAR, V. DAFTARDAR-GEJJI, A predictor-corrector scheme for solving nonlinear delay differential equations of fractional order, Journal of Fractional Calculus and Applications, 1 (5) (2011), $1-9$.

[4] M. BENCHOHRA, M. SAID SOUID, Integrable solutions for implicit fractional order differential equations, TJMM, 6 (2) (2014), 101-107.

[5] K. Diethelm, The analysis of fractional differential equations, Lecture Notes in Mathematics, Springer-Verlag Berlin Heidelberg, 2010.

[6] K. Diethelm, N. J. Ford, Analysis of fractional differential equations, J. Math. Anal. Appl., 265 (2002), 229-248.

[7] J. Dugundji, A. Granas, Fixed Point Theory, Vol-I, Monographic Matematycane, PNW Warsa, 1982.

[8] D. Henry, Geometric theory of semi linear parabolic equations, Springer-Verlag; Berlin, Heidelberge, New York, 1981.

[9] A. A. Kilbas, H. M. SRivastava, J. J. Trujillo, Theory and applications of fractional differential equations, North-Holland Mathematics Studies, vol. 204, Elsevier Science B.V., Amsterdam, 2006.

[10] V. LaKshimikantham, S. LeEla, A Krasnoselskii-Krein-type uniqueness result for fractional differential equations, Nonlinear Analysis, 71 (2009), 3421-3424.

[11] V. LAKSHMiKANTHAM, A. S. VATSALA, Basic theory of fractional differential equations, Nonlinear Analysis, 69 (2008), 2677-2682.

[12] K. S. Miller, B. Ross, An introduction to the fractional calculus and differential equations, John Wiley, New York, 1993.

[13] Juan J. Nieto, Abelghani Ouahab, Venktesh Venktesh, Implicit fractional differential equations via Liouville-Caputo derivative, Mathematics, 3 (2015), 398-411.

[14] S. K. NTOUYAS, Initial and boundary value problems for functional differential equations via the topological transversality method: A survey, Bull. Greek Math. Soc. 40 (1998), 3-41.

[15] I. Podlubny, Fractional differential equations, Academic Press, San Diego, 1999. 